Notfall Rettungsmed 2009 • 12:89-90

DOI 10.1007/s10049-008-1135-4

Online publiziert: 19. Oktober 2009

(c) Springer Medizin Verlag 2009

B. Dirks ${ }^{1} \cdot$ U. Kreimeier ${ }^{2}$

${ }^{1}$ Sektion Notfallmedizin, Klinik für Anästhesiologie, Universitätsklinikum Ulm, Ulm

2 Klinik für Anästhesiologie, Klinikum der Universität München, München

\title{
Vermeintlich harmlose Notfälle: Synkope, Schwindel
}

\section{- Sind Synkope und Schwindel keine Notarztindikationen?}

Diese Fragen werden derzeit intensiv diskutiert und abgewogen. In unserem Fall war die Patientin beim ersten Alarm und Eintreffen des Notarztes ja schon wieder wach.

Schwindel und Synkope werden als Leitsymptome schwerer lebensbedrohlicher Störungen gerade bei älteren Menschen häufig verkannt. Natürlich gibt es viele Patienten, bei denen hinter der Synkope vasovagale Störungen stehen [1], aber beim Patienten mit kardialer Grunderkrankung mag die Synkope auch die letzte Warnung vor dem plötzlichen Herztod sein $[2,3]$. Unbestritten ist, dass der Schwindelattacke häufig ein benigner peripherer paroxysmaler Lagerungsschwindel zu Grunde liegt. Sie mag aber auch eine transitorisch-ischämische Attacke des Hirnstammes sein, der folgende basale Apoplex wird den Patienten lebenslang invalidisieren [4].

Dummerweise sind beide Symptome sehr häufig (Lebenszeitprävalenz von Synkopen $40 \%$, von Schwindel 20-30\%). Es bleibt dem Notfallmediziner in Klinik und Präklinik also nicht erspart, sich mit diesen schillernden Leitsymptomen systematisch auseinander zu setzen, um eine vitale Bedrohung sicher auszuschließen und den Patienten gezielt zuweisen zu können. Schlüssel zur Diagnose ist in beiden Fällen die Anamnese, zumal beim Leitsymptom
Synkope bei Eintreffen des Rettungsdienstes meist keine konkreten Befunde mehr zu erheben sind. Hinzu kommen Grundund Begleiterkrankungen sowie die bestehende Medikation. Gerade letzteres ist in den letzten Jahren durch eine zunehmende Multi-Medikation mit unvorhersagbaren Wechselwirkungen zwischen den einzelnen Wirkstoffen als Akutproblem identifiziert worden ist.

\section{( Eine Abklärung der Grunderkrankung ist zwingend}

Synkopen und Schwindel sind nicht nur häufig und gehen auch nicht selten mit Verletzungen infolge Sturz einher, sie sind für den Betroffenen auch emotional belastend, geben das Gefühl nicht mehr Herr seiner selbst zu sein. Die Abklärung ist nicht selten aufwändig und zeitraubend, häufig findet sich kein pathophysiologisches Korrelat und es bleibt bei der Anfangsdiagose Synkope. Beim nächsten Notarzteinsatz stehen wir dann vor der unangenehmen Frage, ob wir den Patienten zum gleichen diagnostischen Procedere einweisen sollen, das schon erfolglos war. Da Patienten mit Synkope, die gleichzeitig herzkrank sind, aufgrund des Risikos für den plötzlichen Herztod eine schlechte Prognose haben, bleibt dem Notarzt in diesen Fällen nichts anderes übrig, die Abklärung der Grunderkrankung ist zwingend. Beim zweiten Einsatz aus gleicher Ursache sollte man eigentlich 
schon einen Looprecorder beim Patienten vorfinden. Die Frage, ob der (betagte) Patient dann die mögliche Therapie (noch) auf sich nehmen will, muss getrennt bedacht und nach eingehender Aufklärung des Patienten entschieden werden.

Beim Schwindel stehen die Chancen besser. Wenn die korrekte Diagnose gestellt ist, können die meisten Formen peripheren, zentralen und psychogenen Schwindels wirksam behandelt werden. Dennoch, auch diese Patienten können zu Dauerkunden des Notarztes werden.

Es ist also offensichtlich wichtig für Notfallmediziner, sich mit diesen beiden häufigen Symptomen zu beschäftigen. Die Patienten werden es uns danken.

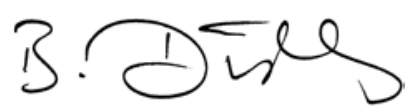

B. Dirks

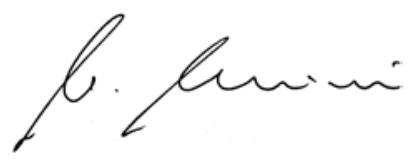

U. Kreimeier

\section{Korrespondenzadresse}

Dr. Dr. B. Dirks

Sektion Notfallmedizin, Klinik für Anästhesiologie, Universitätsklinikum Ulm Prittwitzstr. 43, 89075 Ulm burkhard.dirks@uni-ulm.de

\section{Literatur}

1. Janko S, Hoffmann E (2007) Rhythmogene Synkopen - Wann sind sie prognostisch unbedeutend, wann lebensbedrohlich? Klinikarzt 36:94-99

2. Strupp M, Brandt T (2008) Leitsymptom Schwindel: Diagnose und Therapie. Dtsch Arztebl 105:173-180

3. Sayk F, Schütt M, Dodt C (2009) Rationelle risikoadaptierte Synkopendiagnostik in der Notaufnahme. Notfall Rettungsmed 12 DOI 10.1007/s10049-0081131-8

4. Dennin MA, Haupt WF (2009) Synkope und Schwindel aus neurologischer Sicht. Notfall Rettungsmed 12 DOI 10.1007/s10049-008-1130-9

5. Hoffmann F, Staffler A, Nicolai T, Dalla Pozza R (2009) Management von Synkopen im Kindesund Jugendalter. Notfall Rettungsmed 12 DOI 10.1007/s10049-008-1128-3

\section{P. Luppa, H. Schlebusch (Hrsg.) POCT - Patientennahe Labordiagnostik}

Heidelberg: Springer-Verlag 2008, 382 S., 42 Abb., (ISBN 978-3-540-79151-5), Softcover, 34.95 EUR

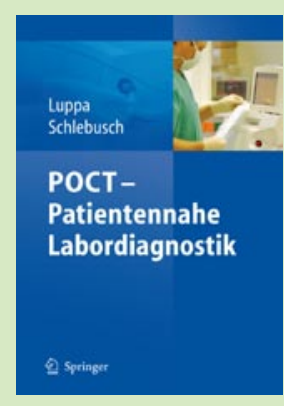

Labordiagnostik am Krankenbett - auch als „Patientennahe Sofortdiagnostik" oder „Point-of-care Testing" (POCT) bezeichnet - wird im klinischen und ambulanten

Bereich zunehmend eingesetzt. Es handelt sich dabei um Untersuchungen, die nicht im Zentrallabor, sondern in unmittelbarer Nähe des Patienten durchgeführt werden. POCT ermöglicht in vielen Bereichen eine schnelle, zuverlässige Diagnostik, die eine zügige Therapieentscheidung unterstützt. Zur POCT-Diagnostik werden anwenderfreundliche Geräte eingesetzt, die auch von Personen ohne spezielle Laborerfahrung bedient werden können. Die korrekte Durchführung der scheinbar einfachen Tests erfordert jedoch eine genaue Kenntnis der testspezifischen Besonderheiten und eine eingehende Schulung des Personals; ferner sind die Richtlinien der Bundesärztekammer zur Qualitätssicherung laboratoriumsmedizinischer Untersuchungen (RiliBÄK), die auch für POCT gelten, zu beachten. Die genannten Maßnahmen sichern die Ergebnisqualität, wodurch inadäquate therapeutische Konsequenzen vermieden werden.

Das Buch gliedert sich in 33 Kapitel und wurde von auf dem jeweiligen Gebiet ausgewiesenen Autoren verfasst. Es ist das erste deutschsprachige Lehrbuch zu diesem Thema.

Besprochen werden:

- Definition und Anwendungsgebiete von POCT, medizinische und wirtschaftliche Bedeutung

- Methodik und analytische Verfahren (Blutglukosebestimmungen, Blutgerinnungsanalytik, Blutgasanalyse, Hämatologie, klinisch-chemische und immunologische Verfahren, Urinanalytik, nicht-invasive Verfahren $u$. a.)
- Organisatorische und ökonomische Aspekte (Implementierung von POCT im Krankenhaus, POCT in der Notfallmedizin und im niedergelassenen Bereich, Vernetzung, Kosten von POCT u.a.)

- Qualitätssicherung (nationale und EUGesetzgebung, Prä- und Postanalytik, Qualitätsmanagement-Systeme, neue Richtlinie der Bundesärztekammer [RiliBÄK 2008], internationale Standardisierung und Akkreditierung u.a.)

- Klinische Anwendungen [Glukosebestimmung und Diabetes-Diagnostik, Gerinnungs-Diagnostik, Diagnostik kardiovaskulärer Erkrankungen (Biomarker zur Vordiagnostik), POCT in der Intensivmedizin, POCT in der Neonatologie, Drogen- und Medikamenten-Screening, mikrobiologische und Urin-Schnelltests u.a.)

- Zukünftige Entwicklungstendenzen Mit diesem Buch werden die Autoren ihrem Anliegen gerecht, auf hohem wissenschaftlichem Niveau ein verständliches und praxisnahes Werk zu bieten. Es bietet eine umfassende Informationsquelle für alle, die POCT bereits einsetzen oder einsetzen wollen, wie beispielsweise Ärzte in Klinik und Praxis, medizinisches Pflegepersonal, MTA, Arzthelferinnen sowie Mitarbeiter im medizinisch-technischen Bereich.

M. Sorger, Bonn 